\title{
Structure of Massive Proto neutron star of PSR J1614-2230 with Trapped Neutrinos
}

\author{
Xia Zhou Huanyu Jia* Bin Hong Xueling Mu \\ School of Physical Science and Technology, Southwest Jiaotong University, Chengdu 610031, \\ China \\ E-mail: hyjiadhome.swjtu.edu.cn
}

\begin{abstract}
Cosmic ray particle acceleration in supernovae is expected to occur. A proto neutron star is formed in the aftermath of the supernova explosion of a massive star. Therefore, the study of properties and structure of proto neutron stars has great implications for investigating the origin and acceleration of cosmic rays. Considering the baryon octet which comprises of the least massive baryons and includes the $\Lambda, \Sigma$ and $\Xi$, focusing on the influence of trapped neutrinos, we study the structure of massive proto neutron star of PSR J1614-2230 in the framework of relativistic mean field theory (RMFT). In this presentation we show the calculation results about constitution, temperature, equation of states, mass and radius of the proto neutron star for different cases of trapped neutrinos. It is found that trapped neutrinos have significant impact on the natures of massive proto neutron star.
\end{abstract}

Keywords Cosmic ray Acceleration, Ptotoneutron star, Trapped Neutrinos, Structure

The 34th International Cosmic Ray Conference,

30 July- 6 August, 2015

The Hague, The Netherlands

${ }^{*}$ Speaker. 


\section{Introduction}

It is generally acknowledged that shock acceleration in supernovae offers a plausible explanation for cosmic ray origin up to energy of $10^{14} \mathrm{eV}[\mathrm{W}$, , [ ] ] X-ray observations of supernova remnants, such as SN1006, have produced evidence of high energy electron acceleration to energies exceeding $10^{13} \mathrm{eV}[\mathrm{B}]$. Although the supernova model for cosmic ray acceleration is appealing from the

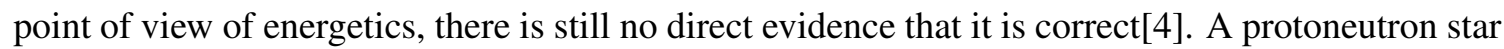
is formed in the aftermath of the supernova explosion of a massive star. Therefore, the study of properties and structure of protoneutron stars has great implication for investigating the origin and acceleration of cosmic rays.

The evolution of the proto neutron star proceeds through several distinct stages which may have various outcomes. The typical neutron star which is one kind of evolution outcomes of a protoneutron star as generally used refers to a cold, neutrino-free star with a mass $\mathrm{M}$ of about $1.5 M_{\odot}$, and the radius $\mathrm{R}$ of $12 \mathrm{~km}[\mathrm{G}]$. But recently, the massive neutron stars have been observed[G, 团 . In particular, the mass of PSR J1614-2230 was measured to be $1.97 \pm 0.04 M_{\odot}$. The equation of state (EOS) and the composition of massive neutron stars aroused researcher's great interest. Many studies have been performed on the composition and global structure of these massive neutron stars with zero temperature. The properties of the protoneutron star corresponding these massive neutron stars, especially PSR J1614-2230 , are of practical importance, but little attention has been on this aspect.

A newly-formed neutron star should accrete its final baryon mass within a second or two of its birth, so that the neutrinos will not have had time to diffuse from the stellar core. These trapped neutrinos may be an important influential factor of the proto neutron star. In this presentation we will discuss the influence of these trapped neutrinos on the structure of the proto neutron star of PSR J1614-2230 in the framework of relativistic mean field theory (RMFT).

\section{Relativistic mean field theory in finite temperature}

When the neutrinos are trapped in the proto neutron star, the composition of the star is determined by the requirement of charge neutrality and chemical equilibrium. The equilibrium condition is

$$
\mu_{i}=b_{i} \mu_{n}-q_{i}\left(\mu_{e}-\mu_{v e}\right)
$$

where $\mu_{i}$ refers to the chemical potential of baryon i, $b_{i}$ is its baryon number and $q_{i}$ is its charge. $\mu_{n}$ , $\mu_{e}$ denote the chemical potentials of neutron and electron, respectively. $\mu_{v e}$ is the chemical potential of the electron neutrino.

In the relativistic mean field theory, baryons B interact via the exchange of mesons. We shall consider the case where the hyperons $\Lambda, \Sigma$ and $\Xi$, are included in the set of baryons. The formulas of these baryons contribution to the equation of state for proto neutron star matter have been listed in references[[1], [13]]. 
For leptons, the interaction is negligible, the partition function of system whose temperature is $\mathrm{T}$ can be written as

$$
\begin{aligned}
& \ln Z_{L}= \frac{V}{T} \sum_{v} \frac{\mu_{v}^{4}}{24 \pi^{2}}\left(1+2\left(\frac{T \pi}{\mu_{v}}\right)^{2}+\frac{7}{15}\left(\frac{T \pi}{\mu_{v}}\right)^{4}\right)+ \\
& \sum_{l}\left(\frac{1}{\pi^{2}} \int_{0}^{\infty} k^{2} d k\left[\ln \left[1+e^{-\left(\varepsilon_{l}-\mu_{l}\right) / T}\right]\right]\right)
\end{aligned}
$$

where $\mathrm{V}$ is the volume.

The first line is the contribution of neutrinos and second line is the contribution from the electron and muon. From this partition function, the number density, energy density and pressure of leptons can be got as follows

$$
\begin{gathered}
\rho_{l}=\frac{1}{\pi^{2}} \int_{0}^{\infty} k^{2} n_{l}(k) d k, \quad \rho_{v}=\frac{\pi^{2} T^{2} \mu_{v}+\mu_{v}^{3}}{6 \pi^{2}} \\
\varepsilon=\sum_{l} \frac{1}{\pi^{2}} \int_{0}^{\infty} \sqrt{\left(k^{2}+m_{l}^{2}\right)} k^{2} n_{l}(k) d k+\sum_{v}\left(\frac{7 T^{4} \pi^{2}}{120}+\frac{T^{2} \mu_{v}^{2}}{12}-\frac{\mu_{v}^{4}}{24 \pi^{2}}\right) \\
P=\frac{1}{3} \sum_{l} \frac{1}{\pi^{2}} \int_{0}^{\infty} \frac{k^{4}}{\sqrt{k^{2}+m_{l}^{2}}} n_{l}(k) d k+\sum_{v} \frac{1}{360}\left(7 T^{4} \pi^{2}+30 T^{2} u_{v}^{2}+\frac{15 u^{4}}{\pi^{2}}\right)
\end{gathered}
$$

Here $l$ denotes electron or muon, $v$ denotes electron-neutrino or muon-neutrino. $n_{l}(k)=\frac{1}{1+e^{\left.\mid \varepsilon_{e}(k)-\mu_{l}\right] / T}}$ is Fermi-Dirac distribution for electron and muon. We can define

$$
Y_{l v}=Y_{l}+Y_{v}=\frac{\rho_{l}+\rho_{v}}{\rho}
$$

as the numbers of leptons per baryon of each flavor of neutrino. Here $l=e$ and $\mu$.

The influence of trapped neutrinos on the PNS can be calculated by fixing relative numbers of leptons in the star. Before that, the coupling parameters between baryons and mesons have to be determined.

\section{Coupling parameters}

The first step in the application of the RMFT is to fix the coupling constants $g_{\sigma}, g_{\omega}, g_{\rho}, \mathrm{b}$ and $\mathrm{c}$ which denotes the coupling strength between nucleons and $\sigma, \omega, \rho$ mesons and meson's self-interaction. These five coupling constants can be fixed by the saturation properties of nuclear matter, in particular, the binding per nucleon $\mathrm{B} / \mathrm{A}$, the saturation baryon density $\rho_{0}$, the effective mass of the nucleon at saturation $m^{*}$, the compression modulus $\mathrm{K}$, and the symmetry energy coefficient $a_{\text {sym }}$. In this study we choose the coupling parameter set GL85[녀]. The hyperon coupling parameters are expressed as ratios to the nucleon couplings

$$
x_{\sigma H}=\frac{g_{\sigma N}}{g_{\sigma H}}, x_{\omega H}=\frac{g_{\omega N}}{g_{\omega H}}, x_{\rho H}=\frac{g_{\rho N}}{g_{\rho H}}
$$


The vector coupling constants for hyperons $x_{\rho H}$ which are determined from SU(6) symmetry constraints [ㄷ]] remain unchanged. They are

$$
x_{\rho \Lambda}=0, x_{\rho \Sigma}=2, x_{\rho \Xi}=1
$$

The scalar meson coupling parameters of hyperons $x_{\sigma H}$ can be calculated from the potential depths $U_{H}^{N}$ of hyperon in the saturated nuclear matter[[0]

$$
U_{H}^{N}=x_{\omega H} V-x_{\sigma H} S
$$

here $S=m-m^{*}, V=\left(\frac{g_{\omega N}}{m_{\omega N}}\right)^{2} \rho_{0}$ are the scalar and vector field strengths for symmetric nuclear matter at saturation. The potential depths $U_{H}^{N}$ of hyperons can be taken as [प], ㅁ, [1], 20, 2], 22] $U_{\Lambda}^{N}=-30 \mathrm{MeV}, U_{\Sigma}^{N}=30 \mathrm{MeV}, U_{\Xi}^{N}=-15 \mathrm{MeV}$.

The masses and radiuses of cold and neutrino-free neutron star can be calculated with $x_{\omega H}$ changing from 0.1 to 1.The calculation results are shown in Fig. 1 and 2.

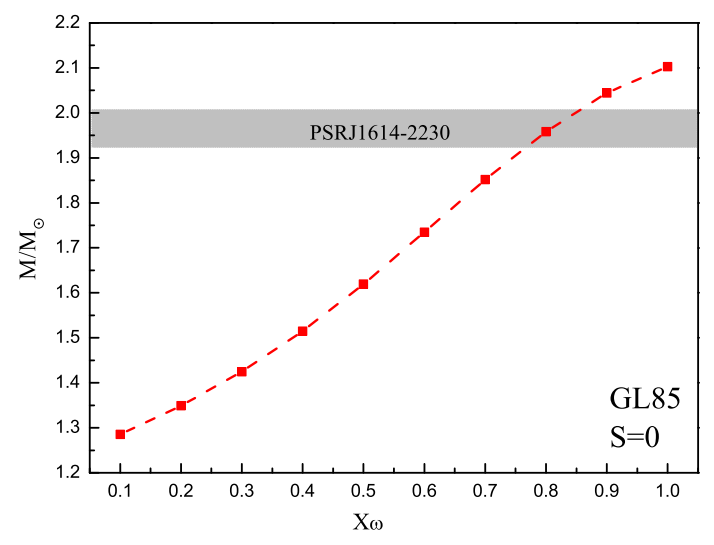

Figure 1: Maxmum neutron star mass as a function of hyperon vector coupling. The shadow area corresponds to the mass of PSR J1614-2230 .

Fig.1 shows that the maximum mass of neutron star increases with $x_{\omega H}$, and when $x_{\omega H}=1.0$, the maximum mass arrives to $2.1 M_{\odot}$ which is larger than the mass of massive neutron star PSR J1614-2230. The calculation result of radius for PSRJ1614-2230 which is $13.03 \mathrm{~km}$ given in Fig. 2 is consistent with the observation for PSR J1614-2230. The above coupling constants with $x_{\omega H}=1.0$ can well describe the structure of PSR J1614-2230. Then we extend these coupling constants to calculate properties of massive protoneutron stars corresponding to PSR J1614-2230. 


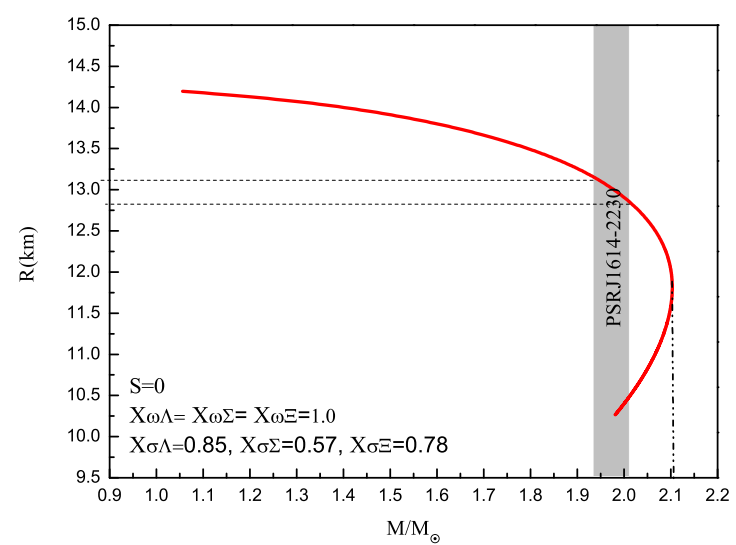

Figure 2: Radius-Mass relationship when $x_{\omega H}=1.0$.

\section{Results and discussion}

For massive PNS, we fix the entropy per baryon $\mathrm{S}=1$ and $Y_{e v}=0.4 . Y_{u v}=0$ because trapped

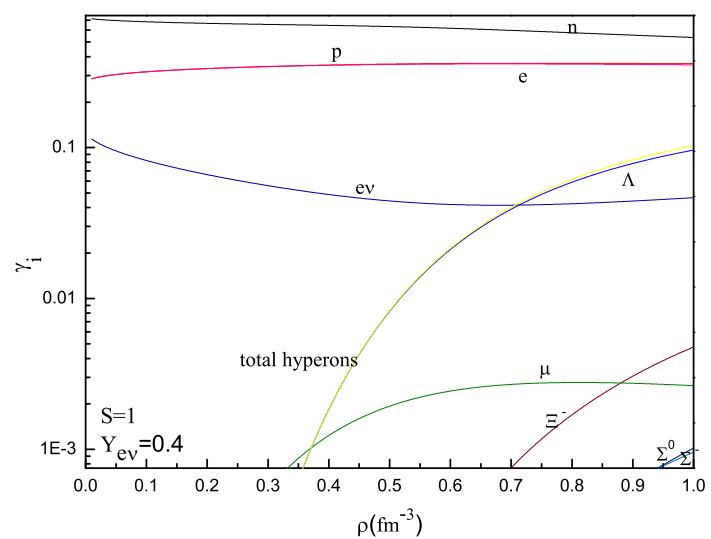

Figure 3: The fraction of various particles in protoneutron star as a function of the total baryon number density when $\mathrm{S}=1$ and $Y_{e v}=0.4$.

neutrinos suppress the appearing of muons, and calculate the fraction of populations of various particles as the total baryon density. The result is shown in Fig.3. From Fig.3 we can see that the fraction of electron neutrino decreases with the total baryon density and gradual increase with the emergence of muon and hyperons.

Fig.4 shows the calculation results of the interior temperature of PRN with different values of $Y_{e v}$. It can be seen that the interior temperature of PRN increase with the baryon density, but the increase rate slow down after hyperons appear. The smaller is the numbers of leptons per baryon of each flavor of neutrino $Y_{e v}$, the lower is the interior temperature of PRN. 


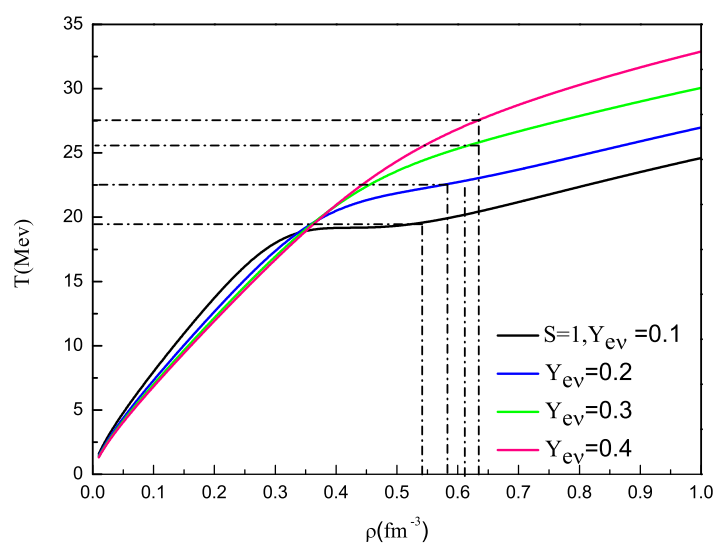

Figure 4: When $\mathrm{S}=1$ and $Y_{e v}$ changes from 0.1 to 0.4 , the interior temperature as the function of total baryon number density.

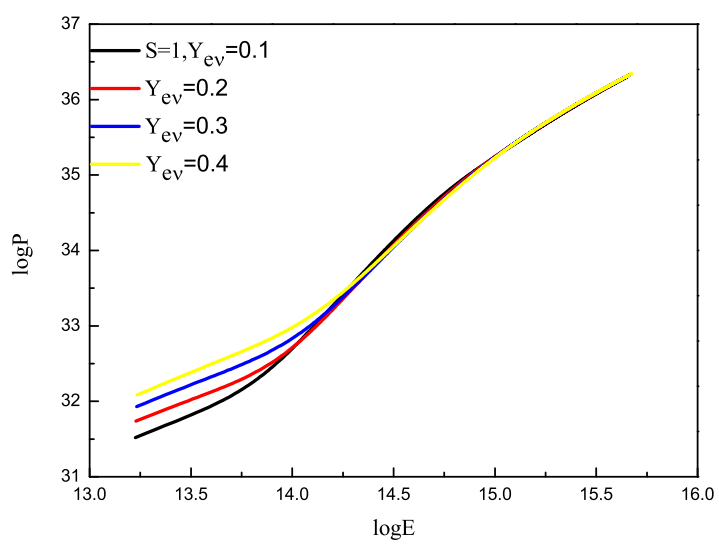

Figure 5: The equations of state of PNS when $Y_{e v}$ changes from 0.1 to 0.4 and $\mathrm{S}=1$.

The equations of state of PNS are shown in Fig. 5 which gives relations between $\log \mathrm{P}$ and $\log \mathrm{E}$ when $Y_{e v}$ changes from 0.1 to 0.4 . We have seen that trapped neutrinos have opposite effect on the equation of state.As to PNS including hyperons, the trapped neutrinos will make the equation of state stiffer as suppress hyperons appearing.With these equations of state, the mass and radius of PNS can be calculated by solving the hydrostatic equilibrium equations of Tolman-OppenheimerVolkoff[223].

The behavior of the radius vs mass is illustrated in Fig.6 for four different neutrino trapped in PNS. From Fig.6 we can get the radius of the protoneutron star corresponding the PSR J1614-2230 whose mass is $1.97 M_{\odot}$. For $Y_{e v}=0.1$, the radius of PNS of PSR J1614-2230 is $13.61 \mathrm{~km}$, whose central density $\rho_{c}$ is $0.54 \mathrm{fm}^{-3}$. The properties of PNS of PSR J1614-2230 for different neutrino trapped are listed in Tab.1. 


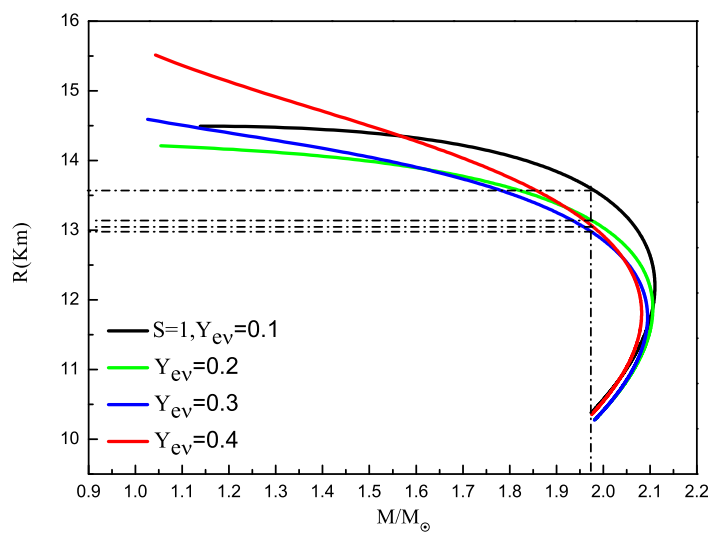

Figure 6: Radius-Mass relationship for $Y_{e v}=0.1 \sim 0.4$ when $\mathrm{S}=1$. The maximum mass reaches up to $2.1 M_{\odot}$. when $Y_{e v}$ is 0.1 .

\begin{tabular}{crrr}
\hline \hline$Y_{e v}$ & $\rho_{c}\left(\mathrm{fm}^{-3}\right)$ & \multicolumn{1}{c}{$T_{c}(\mathrm{MeV})$} & $\mathrm{R}(\mathrm{km})$ \\
\hline 0.1 & 0.54 & 19.57 & 13.61 \\
0.2 & 0.58 & 22.55 & 13.16 \\
0.3 & 0.61 & 25.48 & 13.00 \\
0.4 & 0.63 & 27.47 & 13.09 \\
\hline \hline
\end{tabular}

Table 1: The properties of PNS of PSR J1614-2230 for different neutrino trapped . $\rho_{c}, T_{c}$ and R are central density, central temperature, and radius of PNS of PSR J1614-2230.

\section{Summary}

Basing on the framework of the RMF theory, including the hyperons $\Lambda, \Sigma$ and $\Xi$, we calculate the properties of PNS of PSR J1614-2230 for different neutrino trapped.

It is found that abundance trapped neutrinos lead to fewer hyperons appearing in the massive protoneutron star. The more are neutrinos trapped in the PNS, the higher is the central temperature of PNS. The central temperatures of PNS corresponding to PSR J1614-2230 are 19.57, 22.55, $25.48,27.47 \mathrm{MeV}$ for $Y_{e v}=0.1,0.2,0.3,0.4$. The radius of massive protoneutron star will decrease with increase of $Y_{e v}$. The radius of protoneutron star of PSR J1614-2230 are calculated to 13.61, $13.16,13.00,13.09 \mathrm{~km}$ for $Y_{e v}=0.1,0.2,0.3,0.4$.

These results about the PNS of PSR J1614-2230 may have important significance for studying the neutron star's evolution and acceleration and origin of cosmic rays.

\section{References}

[1] R. Blandfor, D. Eichler, Phys. Rep. 154(1987)1. 
[2] W. I. Axford, Astrophys. J. Suppl. 90(1994)937.

[3] K. Koyama et al. Nature 378(1995)255.

[4] Rene A. ONG, Phys. Rep. 305(1998)93.

[5] J. M. Lattimer and M. Prakash, Science 304, 536 (2004).

[6] P.B.Demorest, T.Pennucci, S.M.Ransom et al, Nature (London) .467, 1081 (2010).

[7] John Antoniadis et al, Science. 340, 1 (2013).

[8] Tsuyoshi Miyatsu, Sachiko Yamamuro, and Ken'ichiro Nakazato, Ap. J. 777, 4 (2013).

[9] S.Weissenborn, D.Chatterjee, J.Schaner-Bielich, Nucl. Phys. A881, 62 (2012).

[10] Isaac Vidana, Nucl. Phys. A914, 367 (2013).

[11] Xian-Feng Zhao, Huan-Yu Jia, Phys. Rev. C 85, 0658069(2012).

[12] ZHANG Hua and JIA Huan-Yu, Commun. Theor. Phys. 45 (2006) pp. 901-905.

[13] M. Prakash, et al. Phys. Rep. 280 (1997) 1.

[14] N.K. Glendenning, Astrophys. J. 293(1985)470.

[15] H. Shen,Phys.Rev. C65, 035802 (2002).

[16] H. Muller,Phys.Rev.C59,1405 (1999).

[17] Millener D J, Dover C B, Gal A. PhRvC, 1988, 38: 2700.

[18] Schaner J, Stoecker H, Greiner C. PhRvC, 1992, 46: 322.

[19] Batty C J, Friedman E, Gal A. PhR, 1997, 287(5): 385.

[20] Schaner-Bielich J, Gal A. PhRvC, 2000, 62: 034311.

[21] Aoki S, Bahk S Y, Chung K S. PhLB, 1995, 355: 45.

[22] Khaustov P, Alburger D E, Barnes P D, et al. PhRvC, 2000, 61: 054603).

[23] Oppenheimer.J.R, Volko.G.M,Phys. Rev. 55, 374(1939). 1. 